$18^{\text {th }}$ European Symposium on Computer Aided Process Engineering - ESCAPE 18

Bertrand Braunschweig and Xavier Joulia (Editors)

(C) 2008 Elsevier B.V./Ltd. All rights reserved.

\title{
Minimization of Life Cycle Greenhouse Emissions and Cost in the Operation of Steam and Power Plants
}

\author{
Pablo E. Martinez and Ana Maria Eliceche ${ }^{\mathrm{a}}$ \\ Chem. Eng. Dept.,Universidad Nacional del Sur-CONICET, Camino La Carrindanga \\ km 7, 8000 Bahía Blanca, Argentina.
}

\begin{abstract}
The main objective of this work is to use environmental and economic objective functions to select the operating conditions of process plants. The operating conditions of a steam and power plant are selected to minimize greenhouse emissions and cost. The battery limits of the utility plant are extended to include the main greenhouse burdens of the imported electricity and its corresponding life cycle from raw material extraction, refining, transport, generation, transmission and waste disposal. Electricity generation by thermoelectric, hydroelectric and nuclear plants is considered. The reduction in $\mathrm{CO}_{2}$ equivalent emissions and its market price is included in the economic objective function. Significant reductions in greenhouse emissions and cost are achieved simultaneously, selecting the operating conditions such as temperature and pressure of high, medium and low pressure steam headers. The operating conditions include discrete decision for the selection of alternative drivers, between electrical motors and steam turbines, and equipment that are on or off, which are represented using binary variables in the mixed integer nonlinear programming problem. Significant reduction in greenhouse emissions and cost are achieved.
\end{abstract}

Keywords: Life Cycle, Greenhouse emissions, Cost, Operation.

\section{Introduction}

The International Panel on Climate Change has strongly recommended the reduction of greenhouse gases emissions as the only way to minimize potentially irreversible impacts on ecosystems and societies. The main source of greenhouse emissions is the combustion of fossil fuels although greenhouse emissions are also present in the entire life cycle of any product or service. $\mathrm{CO}_{2}, \mathrm{~N}_{2} \mathrm{O}, \mathrm{CH}_{4}$ are released from each stage in the supply chain of any product. The upstream processes consume raw materials and energy which have associated greenhouse emissions due to both fossil fuel consumption and fugitive emissions. The upstream processes include raw material extraction, processing and distribution. An exhaustive work on life cycle greenhouse emissions is presented by Weisser (2007) paying special attention to fossil fuel, nuclear and renewable energy technologies in the European Union and Japan. The life cycle approach has been traditionally used to quantify the environmental performance of a product. Azapagic and Clift (1999) have proposed the application of life cycle assessment for the selection of alternative technologies with the e-constraint multiobjective method applied to a mineral processing system. Multi-objective optimization applied to environmental and economic objectives has been treated by authors like Ciric and Huchette (1993) with two objectives, the amount of waste and the net profit of an ethylen glycol production plant. Dantus and High (1999) proposed a method to convert a biobjective optimization 
into a single objective optimization problem; the method proposed is a variation of the utopia point distance minimization, including discrete variables to select the type of reactor to be used in the methyl chloride superstructure plant design.

The steam and power generation system of a petrochemical plant is the sector where fossil fuels are burned to provide utilities to the process plant. This study focuses on the estimation and minimization of life cycle greenhouse emissions and cost of a steam and power plant selecting optimally the operating conditions, solving a multiobjective optimization problem. To assess greenhouse emissions in the life cycle context a key issue is the definition of the life cycle boundaries extending the battery limits of the steam and power sector in order to include the main sources of GHG emissions such as the corresponding to the imported electricity from thermoelectric, hydroelectric and nuclear generation.

Typical operating conditions to be selected in utility systems are temperature and pressures of high, medium and low pressure steam headers, deareator pressure and letdowns flow rates. Binary operating variables are introduced to represent discrete decision such as equipment that can be on or off as boilers and their auxiliary equipment and the selection between optional drivers for pumps that can be either electrical motors or steam turbines. As a case study the steam and power sector of an ethylene plant in operation is analyzed.

\section{Estimation of Greenhouse Emissions}

Greenhouse gases include $\mathrm{CO}_{2}, \mathrm{~N}_{2} \mathrm{O}, \mathrm{CH}_{4}, \mathrm{SF}_{6}$ and CFCs, each of them having different heat-trapping properties. To compare their effects on the atmosphere the Global Warming Potential, the $g w p$ factors are used. Global Worming Potential is the ability of a greenhouse gas to trap heat in the atmosphere relative to an equal amount of carbon dioxide, thus $g w p$ of $\mathrm{CO}_{2}$ has a value of 1 . The next greenhouse gases of importance are $\mathrm{CH}_{4}$ and $\mathrm{N}_{2} \mathrm{O}$ which take values of 21 and 310, over a 100-year time span, Guinée et al (2002), respectively. The emissions of $\mathrm{SF}_{6}$ and CFCs are negligible in fossil fuel combustion and during electricity life cycle (Dones et al, 2004), thus these gases are not considered in the present work. Hence, to obtain the amount of greenhouse emissions $\mathrm{CO}_{2 \mathrm{e}}$ (kilogram of carbon dioxide equivalent per time unit), the $\mathrm{k}$ flow rate is multiplied by its corresponding $g w p_{k}$. In the following sections the evaluation of the greenhouse emissions for the utility plant and the imported electricity are presented.

\subsection{Steam and power generation plant}

The utility sector of the ethylene plant, consume natural gas and a residual gas stream coming from the ethylene plant as fuel to produce high pressure steam. The greenhouse emissions come from the combustion in boilers and a waste heat boiler. The gases produced during combustion contain pollutants like $\mathrm{CO}_{2}, \mathrm{CO}$, NOx, VOCs and trace metals. In this work only the greenhouse emissions are considered.

The combustion emissions for the utility plant (UP) are calculated with the following equation:

$C O_{2 e}^{U P}=\sum_{k}\left[F_{n g} \times \sum_{l} e_{k}^{l}+F_{r g} \times e_{r g, k}\right] \times g w p_{\mathrm{k}} \quad I=1, \ldots, I_{n g}$

Where: $\boldsymbol{F}_{\boldsymbol{n} \boldsymbol{g}}$ is the natural gas flow rate, $\boldsymbol{F}_{\boldsymbol{r} \boldsymbol{g}}$ is the residual gas flow rate, both burned in boilers and waste heat boiler, $\boldsymbol{e}_{\boldsymbol{r g}, \boldsymbol{k}}$ is the residual gas emission factor for pollutant $\boldsymbol{k}$; 
$\boldsymbol{e}_{\boldsymbol{k}}^{\boldsymbol{l}}$ are the emission factor for greenhouse gas $\boldsymbol{k}$ in the life cycle stage $\boldsymbol{I}, \boldsymbol{I}_{\boldsymbol{n}}$ is the total number of life cycle stages, including combustion in the utility plant and the natural gas fuel cycle, for natural gas fuel cycle the extraction and transportation stages are considered. The emission factors for different pollutants $\mathrm{k}$ during natural gas and residual gas combustion are estimated in the following way: stoichiometrically, according to the natural gas composition for $\mathrm{CO}_{2}$ and from AP-42 report (EPA, 1998) for $\mathrm{CH}_{4}$ and $\mathrm{N}_{2} \mathrm{O}$. As the residual gas is produced in the ethylene plant, no life cycle stage has been considered for it.

\subsection{Imported Electricity}

The electricity generation sector in Argentina has contributions from thermoelectric, hydroelectric and nuclear plants. The share of each is seasonal dependent. Thermoelectric power generation consumes coal, oil and natural gas as fuels, nuclear power generation consumes uranium fuel. The greenhouse emissions are estimated with data from National Greenhouse Gases Inventory (2005) including those values for the fossil fuels combustion which are the same values used by IPCC guidance for the country greenhouse emission inventory elaboration. The general equation for estimating the greenhouse emissions in electric power generation includes the following life cycle stages: extraction and processing of raw materials, transport, refining (where it is applicable) and electricity generation itself:

$$
C O_{2 e}{ }^{I E}=\sum_{j} \sum_{k} \sum_{l_{j}} w_{j} \times e_{j k}^{I_{j}} \times g w p_{k}
$$

Where the $\boldsymbol{j}$ subscripts is to accounting for the different way of electricity production, thermoelectric, nuclear and hydroelectric, $\boldsymbol{l}_{\boldsymbol{j}}$ superscript is to accounting for life cycle stage $\boldsymbol{l}$ in electricity generation option $\boldsymbol{j}$ and $\boldsymbol{w}_{\boldsymbol{j}}$ is the amount of electricity imported from each power source option $\boldsymbol{j}$. A detailed analysis of each life cycle stages considered was presented in Eliceche et al (2007a). The methodology was updated with data from Weisser (2007), transmission loss equal to $6 \%$ is also included (EIA, 2003) for all the electricity generation options.

\section{Objective Functions to be minimized}

\subsection{Environmental Objective Function}

The life cycle greenhouse emissions are calculated as the sum of the utility plant greenhouse emissions (Eq. 1) and the imported electricity greenhouse emissions (Eq.2) as follows:

$$
\mathrm{CO}_{2 e}{ }^{\mathrm{LC}}=\mathrm{CO}_{2 e}^{\mathrm{UP}}+\mathrm{CO}_{2 e}^{\mathrm{IE}}
$$

\subsection{Economic Objective Function}

The economic objective function $(C)$ consider: (i) the operating cost including costs of natural gas, freshwater, cooling water treatment and imported electricity as shown in Eliceche et al (2007b) and (ii) income due to greenhouse emissions reduction $\mathbf{C O}_{2 e}{ }^{R}$ traded in the market as shown in the following equation: 
$C=\sum_{i} c_{i} \times F_{i}-C_{2 e}{ }^{R} \times p$

Where the sub index $i$ indicates each term of the operating cost, $\boldsymbol{c}_{\boldsymbol{i}}$ operating cost i, $\boldsymbol{F}_{\boldsymbol{i}}$ represent flows rates and $\boldsymbol{p}$ is the market price of greenhouse emissions in the international emission trade market. A similar objective was used by Hashim et al. (2005) for the optimization of Ontario electricity system including the price of $\mathrm{CO}_{2 \mathrm{e}}$ emissions, solving a linear programming problem.

To select optimally the operating condition of the steam and power sector, both objectives greenhouse emissions and cost are minimized simultaneously as shown in the following section.

\section{Selection of the operating conditions}

Considering the minimization of greenhouse emissions and cost the following multiobjective Mixed Integer Non Linear Programming (MINLP) problem can be formulated:

$$
\begin{array}{cl}
\operatorname{Min}_{x, y} & C(x, y), \mathrm{CO}_{2 e}{ }^{L C}(x, y) \\
\text { s.t.: } & h(x, y)=0 \\
& g(x, y) \leq 0 \\
& x^{L} \leq x \leq x^{U} \\
& x \in R^{n} \\
& y \in\{0.1\}^{m}
\end{array}
$$

Where: $\boldsymbol{C}$ is the economic objective function (Eq. 4); $\boldsymbol{C O}_{2 e}{ }^{\mathbf{L C}}$ are the life cycle greenhouse emissions (Eq. 3); $\boldsymbol{x}$ and $\boldsymbol{y}$ are continuous and binary variables; superscripts $L$ and $U$ indicates lower and upper bounds on the continuous variables. Pressures and temperatures of high, medium and low-pressure steam headers, deaerator pressure and letdowns flow rates are the continuous optimization variables, a subset of vector $\boldsymbol{x}$. Binary variables $\boldsymbol{y}$ represent discrete decisions that allow the selection of alternative pumps drivers such as steam turbines and electrical motors and whether the boilers and their corresponding feed pumps and air fans are on or off. Equality constraints $\boldsymbol{h}(\boldsymbol{x}, \boldsymbol{y})$ represent the steady state modeling of the utility plant, including mass, energy balances and steam properties prediction such as entropy and enthalpy. Inequality constraints $\boldsymbol{g}(\boldsymbol{x}, \boldsymbol{y})$ represent minimum and maximum equipment capacities, logical constraints, operating and design constraints.

The multiobjective (MO) optimization is a system analysis approach to problems with conflictive objectives, a key factor of MO optimization is that rarely exist a single solution that simultaneously optimizes all the objectives. In its place, there is a set of non-inferior solutions where one objective cannot be improved except at expense of another (Ciric et al, 1993). This set of compromise solution corresponds to a set of feasible solutions, generally referred as non-inferior or Pareto optimal solutions. 
Minimimization of Life Cycle Greenhouse Emissions and Cost in the Operation of Steam and Power Plants

\section{Improvements achieved in the operation of the steam and power plant}

A wide variety of multiple objectives techniques exist, a review can be seen in Alves et al (2007). The general approach consists in converting the multiple objectives into a single objective. The numerical results presented in this section have been calculated using as the objective function in problem P1 the sum of $\boldsymbol{C O}_{2 e}{ }^{\mathbf{L C}}$ emissions (Eq. 3) and the economic objective (Eq. 4) as follows:

$$
\mathrm{EnvECO}=\mathrm{CO}_{2 e}{ }^{\mathrm{LC}}+\mathrm{C}
$$

The greenhouse emissions evaluated are $\mathrm{CO}_{2}, \mathrm{~N}_{2} \mathrm{O}$ and $\mathrm{CH}_{4}$. The numerical results correspond to the steam and power sector of an ethylene plant. The MINLP problem P1 is formulated and solved in GAMS (Brooke et al, 2003) using DICOPT as an outerapproximation algorithm, CONOPT algorithm to solve NLP sub problems and CEPLEX algorithm to solve MILP sub problems. Problem P1 was solved in three major iterations and a CPU time of $0.56 \mathrm{sec}$ in a Pentium IV $(1 \mathrm{GHz})$.

The main numerical results on each summand of the objective function EnvEco are reported in Table 1 . The reductions achieved simultaneously are $13 \%$ in $\mathbf{C O}_{2 e}{ }^{L C}$ emissions and $\mathbf{1 8 \%}$ in operating cost. Due to an income in $\mathbf{C O}_{2 e}{ }^{\mathbf{L C}}$ emission trade of $\mathbf{1 4}$ $\%$, the total improvement in the economic performance reaches a $\mathbf{3 2} \%$ with respect to the initial cost.

Table 1. Improvements reached selecting the operating conditions.

\begin{tabular}{lrrrc}
\hline \multicolumn{1}{c}{ Objectives } & \multicolumn{1}{c}{ Units } & Initial point & Solution point & Improvement \% \\
\hline $\mathrm{CO}_{2 e}{ }^{L C}$ & $\mathrm{Kg} \mathrm{CO} 2$ eq./h & 33643.383 & 29290.5500 & $\mathbf{1 2 . 9 4 2}$ \\
Operating Cost & $\mathrm{U} \$ \mathrm{~S} / \mathrm{hr}$ & 981.043 & 803.0862 & $\mathbf{1 8 . 1 4 0}$ \\
$\mathrm{CO}_{2 e}{ }^{L C}$ Income & $\mathrm{U} \$ \mathrm{~S} / \mathrm{hr}$ & 0.000 & 139.2906 & $\mathbf{1 4 . 2 0 0}$ \\
Economic $(\mathrm{C})$ & $\mathrm{U}$ ) $/ \mathrm{hr}$ & 981.043 & 663.7956 & $\mathbf{3 2 . 3 4 0}$ \\
\hline
\end{tabular}

The improvements reported in Table 1 have been achieved selecting the continuous and binary operating conditions of the utility system. Most of the alternative drivers that were electrical motors at the initial point have been switched to steam turbines at the solution point. This is due to the fact that the ratio of $\mathbf{C O}_{2 e}{ }^{\mathbf{L C}}$ emissions per $\mathrm{KW}$ and cost are much smaller in the utility plant than in the imported electricity. The solutions minimizing $\mathbf{C O}_{2 e}{ }^{\mathbf{L C}}$ and cost separately are similar, having a difference in the order of $0.1 \%$ in the respective objective functions. On the other hand minimizing $\mathbf{C O}_{2 e}{ }^{\boldsymbol{U P}}$ (greenhouse emissions of the utility plant only) and cost separately, the solutions differ in the order of $10 \%$. These numerical results indicate that when life cycle boundaries are properly defined, environmental and economic objectives like $\boldsymbol{C O}_{2 e}$ emissions and operating cost are not necessarily conflictive ones, while if the battery limits are used these objectives are likely to render different results. Thus, a key issue when using simultaneously environmental and economic objectives is to define properly the system boundaries, as has been shown in the steam and power sector of an ethylene plant analyzed. The strategy presented can be applied to different steam and power plants. These utility plants are a very important sector of petrochemical plants basically due to the high consumption of non renewable fossil fuels and the combustion emissions generated in boilers. 


\section{Conclusions}

An strategy to select the operating conditions of steam and power plants minimizing greenhouse emissions and cost simultaneously has been presented, solving a mixed integer nonlinear programming problem in GAMS. Significant reductions in the environomic objective can be achieved as shown in Table 1 , reducing $13 \%$ the greenhouse emissions, $18 \%$ the operating cost and an additional $14 \%$ due to $\mathrm{CO}_{2 e}$ emissions trade income that improves the economic objective in more than $30 \%$. Thus a significant improvement is achieved selecting the operating conditions of the utility sector. A proper definition of the life cycle limits is a relevant issue in process optimization when environomic objectives are considered. The numerical results show that objectives like the minimization of greenhouse emissions and cost are not necessarily conflictive ones when the life cycle limits are properly defined.

\section{References}

A. Azapagic and R. Clift, 1999, The application of life cycle assessment to process optimization, Com. \& Chem. Eng., 23, 1509-1526.

A. Brooke, D. Kendrick, A. Meeraus, R. Raman (eds.), 2003, GAMS, A user guide, GAMS Development Corporation, Washington DC.

A. Ciric and S. Huchette, 1993, Multiobjective Optimization Approach to Sensitivity Analysis: Waste Treatment Costs in Discrete process Synthesis and Optimization problems, Ind. Eng. Chem. Res., 32, 2636-2646.

A. Eliceche and P. Martinez, 2007a, Minimization of life cycle CO2 emissions in the operation of a steam and power plant, ESCAPE17, V. Plesu and P.S. Agachi (Editors).

A. Eliceche, S. Corvalan, P. Martinez, 2007b, Environmental life cycle impact as a tool for process optimization of a utility plant, Comp.\& Chem. Eng., 31, 648-656.

D. Weisser, 2007, A guide to life-cycle greenhouse gas (GHG) emissions from electric supply technologies, Energy, 32, 1553-1559.

Energy Information Administration, 2003, Annual energy review. US Department of Energy. Washington, DC, USA. Available from http://www.eia.doe.gov/emeu/aer/contents.html.

H. Hashim, P. Douglas, A. Elkamel and E. Croiset, 2005, Optimization Model for Energy planing with $\mathrm{CO}_{2}$ Emission Consideration, Ind. Eng. Chem. Res., 44, 879-890.

J. Guinée, R. Heijungs, G. Huppes, R. Kleijn, A. Koning, L. van Oers, A. Sleeswijk, S. Suh, H. Udo de Haes (eds.), 2002, Handbook on Life Cycle Assessment. Operational Guide to the ISO Standards. Kluwer Academic Publishers, Dordrecht.

M. Alves and J. Climaco, 2007, A review of interactive methods for multiobjective integer and mixed-integer programming, European J. of Operatinal Research, 180, 99-115.

M. Dantus and K. High, 1999, Evaluation of waste minimization alternatives under uncertainty: a multiobjective optimization approach, Comp. and Chem. Eng., 23, 1493-1508.

National Greenhouse Gases Inventory, 2005, Inventario Nacional de Gases de efecto Invernadero de la Republica Argentina, Fundacion Bariloche, Argentina.

R. Dones, T. Heck and S. Hirschberg, 2004, Greenhouse gas emissions from energy systems, comparison and overview, Encyclopedia Energy, 3, 77-95.

U.S. Environmental Protection Agency, 1998, AP-42, Compilation of air pollutant emission factors, fifth edition, North Carolina. 